\title{
STABILITY OF UNDULOIDAL AND NODOIDAL MENISCI BETWEEN TWO SOLID SPHERES
}

\author{
BORIS RUBINSTEIN AND LEONID FEL
}

Communicated by Jean-Francois Ganghoffer

Abstract. We find the existence conditions of unduloidal and nodoidal menisci between two solid spheres and study their stability under axisymmetric perturbations in the framework of non-spectral theory of stability of axisymmetric menisci between two axisymmetric solid bodies in the absence of gravity.

MSC: 53A10, 76B45

Keywords: axisymmetric pendular rings, inflection points, stability problem

\section{Contents}

$\begin{array}{lll}1 & \text { Introduction } & 78\end{array}$

2 Axisymmetric Menisci Between Solid Bodies and Their Existence 80

3 Existence of Axisymmetric Menisci Between Two Spheres 84

3.1 Constraints of $\mathbf{A}$ and $\mathbf{B}$ Types . . . . . . . . . . . . . . . . . . . . 84

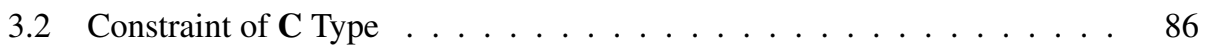

3.3 Constraint of $\mathbf{D}$ Type . . . . . . . . . . . . . . . . . 87

4 Menisci Between Equal Spheres. Face-to-Face Setup 87

4.1 Unduloidal Menisci Between Two Solid Spheres . . . . . . . . . . . . . 88

4.2 Nodoidal Menisci Between Solid Spheres (2 Types of Constraints) . . . . 89

4.3 Nodoidal Menisci Between Solid Spheres (3 Types of Constraints) . . . . 89

4.4 Menisci Between Two Equal Contacting Spheres . . . . . . . . . . . . . 90

5 Menisci Between Equal Spheres. Back-to-Back Setup 93

6 Menisci Between Equal Spheres. Face-to-Back Setup 94

7 Menisci Between Nonequal Spheres 95

7.1 Face-to-Face Setup . . . . . . . . . . . . . . . . . . . . . 95

7.2 Face-to-Back Setup . . . . . . . . . . . . . . . . . 96

$\begin{array}{ll}\text { References } & 97\end{array}$

$\begin{array}{ll}\text { doi: } 10.7546 / j g s p-39-2015-77-98 & 77\end{array}$ 


\section{Introduction}

Pendular rings (PR) in the absence of gravity between two axisymmetric solid bodies (SB) with free contact lines (CL) are surfaces of revolution with constant mean curvature (CMC) classified by Delaunay in [2]: cylinder (Cyl), sphere (Sph), catenoid (Cat), nodoid (Nod) and unduloid (Und). Two questions are important in this regard: what is an exact shape (meniscus) of PR in the given setup and how stable is it. The first question would be answered once one could find a solution of the Young-Laplace equation (YLE) supplemented by boundary conditions (BC) of free CL and given PR volume? Recent progress [11] in the PR problem has shown the existence of multiple solutions of YLE for given PR volume and as a consequence poses a question on menisci stability as a menisci selection rule.

There are two different approaches to study stability of PR between two SB with free CL. The first approach was initiated by Vogel $[14,15]$ and based on the study of the Sturm-Liouville equation (SLE) and its spectrum. Implementation of this approach is a difficult task: only several exact results for Cat [21], Sph [12] and Und (with special contact angle values) [4,15] between two plates are known. Investigation of menisci between other surfaces encounters even more difficulties of finding analytically a spectrum of SLE with given shape of SB (Cyl [16] and convex Und and Nod between equal spheres [17, 19]).

Another approach was suggested recently [3] as a part of variational problem with minimized and constrained functionals and free endpoints moving along two given planar curves $S_{1}, S_{2}$. It is based on Weierstrass' formula of second variation $\delta^{2} W$ for isoperimetric problem. A freedom of endpoints allows to derive $\delta^{2} W$ as a quadratic form in perturbations $\delta \phi_{j}$ of the endpoints $\phi_{j}$ along $S_{j}\left(\psi_{j}\right)$

$$
\delta^{2} W=Q_{11}\left(\delta \psi_{1}\right)^{2}+2 Q_{12} \delta \psi_{1} \delta \psi_{2}+Q_{22}\left(\delta \psi_{2}\right)^{2}, \quad Q_{i j}=Q_{i j}\left(\phi_{2}, \phi_{1}\right)
$$

and find in the plane $\left\{\phi_{1}, \phi_{2}\right\}$ a stability domain Stab where $\delta^{2} W \geq 0$ (see Theorem 4.1 in [3]). Stability of menisci between parallel plates under axisymmetric perturbations were studied in [3] for all Delaunay's surfaces. We also have found Stab for Cat and Cyl between two SB: spheres, paraboloids, catenoids, ellipsoids and between sphere and plane. This approach has no limitations to find Stab analytically for arbitrary meniscus and SB shapes.

The present paper deals with a more difficult case when Und and Nod menisci are trapped between solid spheres. Compared with menisci geometry between two plates, this problem leads to a question of menisci existence determined by Und and Nod geometry between two spheres. Thus, we have to consider the stability Stab and existence Exst domains such that Stab $\subseteq$ Exst and in order to establish 
the latter we need a simple analytical geometry. We consider three different setups of semispheres (face and back) where the meniscus approaches the spheres: face-to-face (F-F), face-to-back (F-B) and back-to-back (B-B). In [3] there were



a)

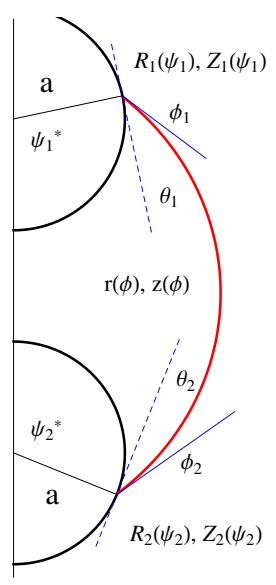

b)

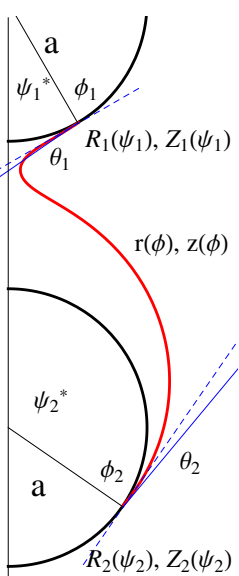

c)

Figure 1. (Color online) Sketches (meridional Sections) of three menisci between two equal spheres of radius $a$ showing the contact angles $\theta_{1}, \theta_{2}$, filling angles $\psi_{1}^{*}, \psi_{2}^{*}$ and coordinates of the endpoints $\phi_{1}, \phi_{2}$ : a) concave meniscus, F-F setup, b) convex meniscus, B-B setup, c) meniscus with one inflection point, F-B setup.

elaborated the main tools (Theorem 4.1) and formulas (Sections 5 and 6) for calculating the stability domain Stab. Therefore, in the present paper the results are mostly presented by figures which content is similar to those presented in [3] for less difficult setups of menisci between SB.

The paper is organized in seven sections. In Section 2 we consider four different types of constraints which define the existence of menisci between two convex SB (not necessarily spheres), and derive the conditions when they occur. In Section 3 we specify them for the case of two solid spheres; we discuss their coexistence and establish Exst domain in different setups. In Sections 4, 5 and 6, based on Theorem 4.1 in [3], we give a detailed analysis of Stab domains for menisci between equal spheres with F-F, B-B and F-B setups, respectively, and find stable Und with two inflection points (IP). In Section 7 we show how a non-equivalence of the spheres affects both Stab and Exst domains. 


\section{Axisymmetric Menisci Between Solid Bodies and Their Existence}

Consider axisymmetric PR between two SB in absence of gravity. The axial symmetry of SB is assumed along $z$-axis (see Fig. 1). The shapes of meniscus $\{r(\phi), z(\phi)\}$ and two SB $\left\{R_{j}\left(\psi_{j}\right), d_{j}+Z_{j}\left(\psi_{j}\right)\right\}$ are given in cylindrical coordinates. The filling angle $\psi_{j}$ along the $j$-th solid-liquid interface satisfies $0 \leq \psi_{j} \leq \infty$ for unbounded SB and $0 \leq \psi_{j}<\infty$ for bounded SB.

Functions $r(\phi)$ and $z(\phi)$ are defined in the range $\phi_{2} \leq \phi \leq \phi_{1}$ and satisfy YLE with curvature $H$

$$
S_{H}=\frac{z^{\prime}}{r\left(r^{2}+z^{2}\right)^{1 / 2}}+\frac{z^{\prime \prime} r^{\prime}-z^{\prime} r^{\prime \prime}}{\left(r^{\prime 2}+z^{\prime 2}\right)^{3 / 2}}
$$

where $S_{H}= \pm 1$ corresponds to the menisci with positive and negative curvature $H$, respectively. Equation (2) is supplemented by Young (transversality) relations for given contact angles $\theta_{j}$

$$
\theta_{j}=(-1)^{j-1}\left(\arctan \frac{z^{\prime}\left(\phi_{j}\right)}{r^{\prime}\left(\phi_{j}\right)}-\arctan \frac{Z^{\prime}\left(\psi_{j}^{*}\right)}{R^{\prime}\left(\psi_{j}^{*}\right)}\right), \quad j=1,2, \quad \theta_{j} \geq 0
$$

and consistency equalities

$$
\begin{array}{ll}
z\left(\phi_{1}\right)=d_{1}+Z_{1}\left(\psi_{1}^{*}\right), & r\left(\phi_{1}\right)=R_{1}\left(\psi_{1}^{*}\right) \\
z\left(\phi_{2}\right)=d_{2}+Z_{2}\left(\psi_{2}^{*}\right), & r\left(\phi_{2}\right)=R_{2}\left(\psi_{2}^{*}\right)
\end{array}
$$

where $d=d_{1}-d_{2}$ is the distance between centers of $S_{1}$ and $S_{2}$. Throughout this paper we use a standard parameterization [3] for menisci with $H \neq 0$ which goes back to [6] and [9, pp.72-73]

$$
\begin{aligned}
& r(\phi)=\sqrt{1+B^{2}+2 B \cos \left(S_{H} \phi\right)} \\
& z(\phi)=M\left(S_{H} \phi, B\right)-M\left(S_{H} \phi_{2}, B\right)+Z_{2}\left(\psi_{2}^{*}\right) \\
& M(\phi, B)=(1+B) E(\phi / 2, m)+(1-B) F(\phi / 2, m), \quad m=2 \sqrt{B} /(1+B)
\end{aligned}
$$

where $F(x, m)$ and $E(x, m)$ denote elliptic integrals of the first and the second kind. Formulas (5) describe four Delaunay's surfaces with nonzero curvature $H$ : Cyl, $B=0$, Und, $0<B<1$, Sph, $B=1$ and Nod, $B>1$.

The choice of parameterization (5) was dictated in [3] by convenience to represent the stability domain Stab in the plane $\left\{\phi_{1}, \phi_{2}\right\}$. It differs from those in $[1,5,10$, $11,18]$, although the elliptic integrals make all them members of the same family. In the last decade a conformal parameterization of Delaunay surfaces remains an area of active research $[7,8,13]$. 
Regarding (5), we assume that in the range $\phi_{2}<\phi<\phi_{1}$ the ordinate $z(\phi)$ is a growing function $z\left(\phi_{2}\right)<z(\phi)<z\left(\phi_{1}\right)$. According to (5) we get

$$
\Delta\left(\phi_{1}, \phi_{2}, S_{H}, B\right)=M\left(S_{H} \phi_{1}, B\right)-M\left(S_{H} \phi_{2}, B\right)>0
$$

that determines $S_{H}$ introduced in (2). This value cannot be defined when $z\left(\phi_{1}\right)=$ $z\left(\phi_{2}\right)$ for $\phi_{1} \neq \phi_{2}$. The condition (6) implies that all unduloids have positive curvature, i.e., $S_{H}=1$. It follows from the explicit expression that $z^{\prime}(\phi)=$ $S_{H}\left(1+B \cos \left(S_{H} \phi\right)\right) / r$, leading to positive $z^{\prime}(\phi)$ for $B<1$.

Once $r(\phi)$ and $z(\phi)$ are parameterized by (5) we have to determine the PR existence as a physically valid object. This leads to restriction on parameters $B, \phi_{1}$, $\phi_{2}$, important for nonplanar SB and makes the stability domain Stab substantially dependent on conditions of PR existence. This phenomenon was observed in [3] for Cat between two spheres and also announced in [19] for Nod between equal spheres with contact angles $90^{\circ} \leq \theta_{j}<180^{\circ}$. In other words, a meniscus geometry has to satisfy requirements on $B, \phi_{1}, \phi_{2}$ to avoid different types of meniscus nonexistence which can be distributed into four major types.

- Type A: meniscus does not reach solid surface, Fig. 2a.

This condition is applicable only to SB with finite maximal radial size $R_{\max }$

$$
1+B^{2}+2 B \cos \phi \geq R_{\max }^{2}
$$

- Type B: meniscus reaches solid surface with negative contact angle, Figs. $2 \mathrm{~b}$.

Let PR be trapped between two SB and let a contact angle $\theta_{2}$ at $S_{2}$ be given. Consider $S_{1}$ and require $\theta_{1} \geq 0$, otherwise the meniscus "pierces" $S_{1}$ and contacts it from "inside". The critical endpoint $\phi_{1}^{s}$ corresponding to $\theta_{1}=0$ satisfies equalities:

$$
\begin{aligned}
z^{\prime}\left(\phi_{1}^{s}\right) / r^{\prime}\left(\phi_{1}^{s}\right) & =Z_{1}^{\prime}\left(\psi_{1}^{*}\right) / R_{1}^{\prime}\left(\psi_{1}^{*}\right), \quad r\left(\phi_{1}^{s}\right)=R_{1}\left(\psi_{1}^{*}\right) \\
z\left(\phi_{1}^{s}, \phi_{2}\right) & =d_{1}+Z_{1}\left(\psi_{1}^{*}\right) .
\end{aligned}
$$

For given $B$ we have to find $\phi_{1}^{s}, \phi_{2}, \psi_{1}^{*}, \psi_{2}^{*}$ and locations $d_{j}$ of SBs on $z$ axis. Choose a reference frame in such a way that $z\left(\phi_{2}\right)=0$. According to (3-5), we have $z(\phi)=M\left(S_{H} \phi, B\right)-M\left(S_{H} \phi_{2}, B\right)$. Thus, solving another three equations

$$
Z_{2}\left(\psi_{2}^{*}\right)=-d_{2}, \quad r\left(\phi_{2}\right)=R_{2}\left(\psi_{2}^{*}\right), \quad \theta\left(\phi_{2}, \psi_{2}^{*}\right)=\theta_{2}
$$

we find $\psi_{2}^{*}, \phi_{2}$ and $d_{2}$ as explicit (or implicit) expressions. Resolving now the two first equations in (8) w.r.t. $\phi_{1}^{s}$ and $\psi_{1}^{*}$ we find them also as explicit (or implicit) expressions. 


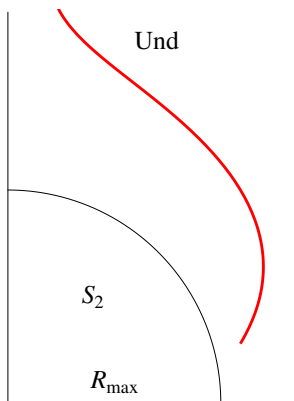

a)

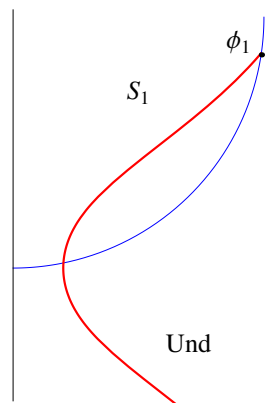

b)

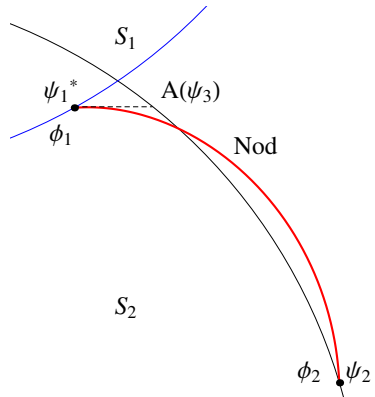

c)

Figure 2. (Color online) Sketches of menisci which have no physical meaning due to the different reasons: a) meniscus does not reach the solid surface $S_{2}$, b) meniscus reaches solid surface $S_{1}$ with negative contact angle and c) meniscus reaches $S_{1}$ at the endpoint which is immersed in $S_{2}$.

The shift $d_{1}$ follows from the third equation in (8), $d_{1}=z\left(\phi_{1}^{s}, \phi_{2}\right)-Z_{1}\left(\psi_{1}^{*}\right)$. The computation of $\phi_{1}^{s}$ and $\psi_{1}^{*}$ can be performed as follows. First, note that $r r^{\prime}=$ $B \sin \left(S_{H} \phi\right)$, and $r z^{\prime}=1+B \cos \left(S_{H} \phi\right)$. From (5) we obtain $2 B \cos \left(S_{H} \phi_{1}^{s}\right)=$ $R^{2}\left(\psi_{1}^{*}\right)-1-B^{2}$, and find

$$
2 B \sin \left(S_{H} \phi_{1}^{s}\right)= \pm \sqrt{\left[R^{2}\left(\psi_{1}^{*}\right)-(1-B)^{2}\right]\left[(1+B)^{2}-R^{2}\left(\psi_{1}^{*}\right)\right]}
$$

where the sign is determined by the value of $\phi_{1}^{s}$. The first equation in (8) reads

$$
\pm \frac{Z_{1}^{\prime}\left(\psi_{1}^{*}\right)}{R_{1}^{\prime}\left(\psi_{1}^{*}\right)} \sqrt{\left[R^{2}\left(\psi_{1}^{*}\right)-(1-B)^{2}\right]\left[(1+B)^{2}-R^{2}\left(\psi_{1}^{*}\right)\right]}=R^{2}\left(\psi_{1}^{*}\right)+1-B^{2}
$$

and it should be resolved w.r.t. $\psi_{1}^{*}$ in the prescribed range of the values of $\psi_{1}$. Substitution of this value $\psi_{1}^{*}$ into condition $2 B \cos \left(S_{H} \phi_{1}^{s}\right)=R^{2}\left(\psi_{1}^{*}\right)-1-B^{2}$, allows to compute $\phi_{1}^{s}$. Similarly one can obtain the relation describing the condition $\theta_{2}=0$.

After obtaining the value $\phi_{j}^{s}$ one has to check if the meniscus arrives at the corresponding $\mathrm{SB}$ is indeed outside of the $\mathrm{SB}$. To do this introduce $z^{*}=z\left(\phi_{j}^{s}\right)+$ $\delta z_{j}, \delta z_{j}=(-1)^{j} \delta z$, such that also $Z_{j}^{*}=Z_{j}\left(\psi_{j}^{*}\right)+\delta z_{j}$. Writing

$$
z\left(\phi_{j}^{s}+\delta \phi_{j}\right)=z\left(\phi_{j}^{s}\right)+\delta z_{j}, \quad Z_{j}\left(\psi_{j}^{*}+\delta \psi_{j}\right)=Z_{j}\left(\psi_{j}^{*}\right)+\delta z_{j}
$$

express $\delta \psi_{j}, \delta \phi_{j} \ll 1$ in the linear approximation $\delta \phi_{j}=\delta z_{j} / z^{\prime}\left(\phi_{j}^{s}\right), \delta \psi_{j}=$ $\delta z_{j} / Z_{j}^{\prime}\left(\psi_{j}^{*}\right)$. Write the radial coordinates of the meniscus and the SB at $z=z^{*}$

$$
\begin{aligned}
r\left(\phi_{j}^{s}+\delta \phi_{j}\right) & =r\left(\phi_{j}^{s}\right)+r^{\prime}\left(\phi_{j}^{s}\right) \delta \phi_{j}+r^{\prime \prime}\left(\phi_{j}^{s}\right) \delta \phi_{j}^{2} / 2 \\
R_{j}\left(\psi_{j}^{*}+\delta \psi_{j}\right) & =R_{j}\left(\psi_{j}^{*}\right)+R_{j}^{\prime}\left(\psi_{j}^{*}\right) \delta \psi_{j}+R_{j}^{\prime \prime}\left(\psi_{j}^{*} s\right) \delta \psi_{j}^{2} / 2 .
\end{aligned}
$$


Calculate the difference

$$
r\left(\phi_{j}^{s}+\delta \phi_{j}\right)-R_{j}\left(\psi_{j}^{*}+\delta \psi_{j}\right)=\left[\frac{r^{\prime \prime}\left(\phi_{j}^{s}\right)}{z^{\prime 2}\left(\phi_{j}^{s}\right)}-\frac{R_{j}^{\prime \prime}\left(\psi_{j}^{*}\right)}{Z_{j}^{\prime 2}\left(\psi_{j}^{*}\right)}\right] \frac{\delta z_{j}^{2}}{2}
$$

which sign is defined by the expression in the square brackets. As the meniscus is outside of the SB when this difference is positive we obtain by substituting (5) into (11) the following condition

$$
\delta \rho=\frac{r^{\prime \prime}\left(\phi_{j}^{s}\right)}{z^{\prime 2}\left(\phi_{j}^{s}\right)}-\frac{R_{j}^{\prime \prime}\left(\psi_{j}^{*}\right)}{Z_{j}^{\prime 2}\left(\psi_{j}^{*}\right)}=-\frac{r^{2}\left(\phi_{j}^{s}\right) B \cos \phi_{j}^{s}+B^{2} \sin ^{2} \phi_{j}^{s}}{r\left(\phi_{j}^{s}\right)\left(1+B \cos \phi_{j}^{s}\right)^{2}}-\frac{R_{j}^{\prime \prime}\left(\psi_{j}^{*}\right)}{Z_{j}^{\prime 2}\left(\psi_{j}^{*}\right)}>0
$$

or its equivalent

$$
\delta \rho^{*}=\frac{r^{\prime \prime}\left(\phi_{j}^{s}\right)}{r^{\prime 2}\left(\phi_{j}^{s}\right)}-\frac{R_{j}^{\prime \prime}\left(\psi_{j}^{*}\right)}{R_{j}^{\prime 2}\left(\psi_{j}^{*}\right)}=-\frac{1}{r\left(\phi_{j}^{s}\right)}\left[1+\frac{r^{2}\left(\phi_{j}^{s}\right) B \cos \phi_{j}^{s}}{B^{2} \sin ^{2} \phi_{j}^{s}}\right]-\frac{R_{j}^{\prime \prime}\left(\psi_{j}^{*}\right)}{R_{j}^{\prime 2}\left(\psi_{j}^{*}\right)}>0 .
$$

The derived conditions $(12,13)$ are particular cases of a more general case when the meniscus is partially immersed into SB.

- Type C: meniscus reaches one $S B$ at the endpoint which is immersed into the other SB, Fig. 2c.

Let a lower of two intersecting SB be "pierced" by meniscus. Choose a reference frame in such a way that $z\left(\phi_{2}\right)=d_{2}+Z_{2}\left(\psi_{2}^{*}\right)=0$. A point $A\left(\psi_{3}\right) \in S_{2}$ is located at $\left\{R_{2}\left(\psi_{3}\right), d_{2}+Z_{2}\left(\psi_{3}\right)=z\left(\phi_{1}\right)\right\}$ where $z\left(\phi_{1}\right)=M\left(S_{H} \phi_{1}, B\right)-M\left(S_{H} \phi_{2}, B\right)$. The meniscus does not exist if $R_{2}\left(\psi_{3}\right)>R_{1}\left(\psi_{1}^{*}\right)=r\left(\phi_{1}\right)$. Summarizing necessary formulas we arrive at requirements of meniscus nonexistence

$$
Z_{2}\left(\psi_{3}\right)-Z_{2}\left(\psi_{2}^{*}\right)=\Delta\left(\phi_{1}, \phi_{2}, S_{H}, B\right), R_{2}\left(\psi_{2}^{*}\right)=r\left(\phi_{2}\right), R_{2}\left(\psi_{3}\right)>r\left(\phi_{1}\right) .
$$

Using an invariance of nonexistence phenomenon under permutation of the upper and lower SB write the requirements of meniscus nonexistence when an upper of two intersecting SB is "pierced" by meniscus

$Z_{1}\left(\psi_{3}\right)-Z_{2}\left(\psi_{1}^{*}\right)=-\Delta\left(\phi_{1}, \phi_{2}, S_{H}, B\right), R_{1}\left(\psi_{1}^{*}\right)=r\left(\phi_{1}\right), R_{1}\left(\psi_{3}\right)>r\left(\phi_{2}\right)$.

- Type D: the center of $S_{2}$ is above the center of $S_{1}$.

This leads to meniscus that reaches $S_{1}$ at the endpoint which is immersed in $S_{2}$ and reaches $S_{2}$ at the endpoint which is immersed in $S_{1}$. To find the restricting relation make use of (3) and eliminate there $\psi_{j}^{*}$. Thus, we arrive at the restricting relation $d_{1}=d_{2}$

$$
z_{1}\left(\phi_{1}\right)-z_{2}\left(\phi_{2}\right)=\Delta\left(\phi_{1}, \phi_{2}, S_{H}, B\right)=Z_{1}\left(\psi_{1}^{*}\right)-Z_{2}\left(\psi_{2}^{*}\right), \quad \psi_{j}^{*}=R_{j}^{-1}\left[r_{j}\left(\phi_{j}\right)\right]
$$

where $f^{-1}$ denotes the inverse function of $f$. 


\section{Existence of Axisymmetric Menisci Between Two Spheres}

In this section we specify formulas (7-16) for two solid spheres with radius $a$ given by the formulas

$$
R_{j}\left(\psi_{j}\right)=a \sin \psi_{j}, \quad Z_{j}\left(\psi_{j}\right)=(-1)^{j} a \cos \psi_{j} .
$$

\subsection{Constraints of A and B Types}

There exists a critical angle $\phi_{A}$ related to the menisci nonexistence of type $\mathbf{A}$ (see Fig. 2a). It corresponds to a meniscus which does not reach a solid sphere

$$
a^{2}=1+B^{2}+2 B \cos \phi_{A} \quad \rightarrow \quad \cos \phi_{A}=\left(a^{2}-1-B^{2}\right) / 2 B .
$$

A critical angle $\phi_{B}$ of the type $\mathbf{B}$ (Fig. 2b) may be calculated using relations

$$
\frac{R^{\prime}\left(\psi_{B}^{*}\right)}{Z^{\prime}\left(\psi_{B}^{*}\right)}=\frac{r^{\prime}\left(\phi_{B}^{*}\right)}{z^{\prime}\left(\phi_{B}^{*}\right)}, \quad r\left(\phi_{B}\right)=R\left(\psi_{B}^{*}\right), \quad z\left(\phi_{B}\right)=d+Z\left(\psi_{B}^{*}\right)
$$

in (5) and (17). Then we obtain for menisci with positive curvature $\left(S_{H}=1\right)$

$$
\tan \psi_{B}^{*}=\mp \frac{1+B \cos \phi_{B}}{B \sin \phi_{B}}, \quad \sin \psi_{B}^{*}=\frac{\sqrt{1+B^{2}+2 B \cos \phi_{B}}}{a}
$$

where $+/-$ sign corresponds the lower (upper) sphere. Eliminating of $\psi_{B}^{*}$ from (20) we obtain

$$
\cos \phi_{B}=-\frac{1+B^{2}+b}{B(2+b)}, \quad b= \pm a .
$$

When $2+b>0$, one can represent (21) as follows

$$
B(2+b)>1+B^{2}+b>-B(2+b) \rightarrow\left\{\begin{array}{l}
(1-B)(1-B+b)<0 \\
(1+B)(1+B+b)>0 .
\end{array}\right.
$$

In case of Und we have a negative $b=-a$

$$
B<1, \quad-2<b<B-1, \quad-B-1<b \rightarrow 1-B<a<1+B .
$$

In case of convex Nod we have a positive $b=a$

$$
B>1, \quad b>B-1>-B-1 \rightarrow a>B-1 .
$$

When $2+b<0$, one can represent (21) as follows

$$
-B(2+b)>1+B^{2}+b>B(2+b) \rightarrow\left\{\begin{array}{l}
(1-B)(1-B+b)>0 \\
(1+B)(1+B+b)<0 .
\end{array}\right.
$$


In case of Und we have a negative $b=-a$

$$
B<1, \quad B-1<b<-1-B, \quad b<-2 \rightarrow 2<a<1-B
$$

which is a contradiction. In case of convex Nod we have a negative $b=-a$

$$
B>1, \quad b<-B-1<B-1, \quad b<-2, \quad \rightarrow \quad a>B+1 .
$$

We have to make certain that all menisci have a physical meaning. Namely, we require that the menisci approaching contact point on the sphere with $\phi_{B}$ given by (21) are outside of the sphere. As $R_{j}^{\prime \prime}\left(\psi_{j}^{*}\right) / Z_{j}^{\prime 2}\left(\psi_{j}^{*}\right)=-1 / R_{j}\left(\psi_{j}^{*}\right)=-1 / r\left(\phi_{j}^{s}\right)$, using the condition (12) we find

$$
\begin{aligned}
r\left(\phi_{B}\right) \delta \rho & =1-\frac{B \cos \phi_{B}\left(1+B^{2}+2 B \cos \phi_{B}\right)+B^{2} \sin ^{2} \phi_{B}}{\left(1+B \cos \phi_{B}\right)^{2}} \\
& =\frac{1-B^{2}}{1+B \cos \phi_{B}}=2 \mp a
\end{aligned}
$$

where the "+" sign is selected for Nod in (23), and the "-" sign stands for Und in (22) and Nod in (25). In the last case $a>2$, so that the Nod meniscus in (25) approaches the contact point immersed into the sphere and thus it should be removed from further consideration.

Summarize $(22,23)$. The menisci exist when

$$
\begin{aligned}
& \text { Und : }\left\{\begin{array} { l } 
{ B < 1 } \\
{ | a - 1 | < B , }
\end{array} \left\{\begin{array}{l}
\cos \phi_{B}=-\frac{1+B^{2}-a}{B(2-a)} \\
1+B \cos \phi_{B}=\frac{1-B^{2}}{2-a}
\end{array}, \tan ^{2} \psi_{B}^{*}=\frac{1-B^{2}}{B^{2}-(a-1)^{2}}\right.\right. \\
& \text { Nod : }\left\{\begin{array}{l}
B>1 \\
a+1>B,
\end{array},\left\{\begin{array}{l}
\cos \phi_{B}=-\frac{1+B^{2}+a}{B(2+a)} \\
1+B \cos \phi_{B}=\frac{1-B^{2}}{2+a}
\end{array}, \tan ^{2} \psi_{B}^{*}=\frac{B^{2}-1}{(a+1)^{2}-B^{2}}\right.\right.
\end{aligned}
$$

A choice of the sign of $\tan \psi_{B}^{*}$ is dictated by the value of $\phi_{B}$ running in the range $[0,2 \pi]$. To choose a correct sign introduce for the upper and lower spheres two variables $\sigma_{1}$ and $\sigma_{2}$, respectively. The ranges $0 \leq \psi_{B}^{*} \leq \pi / 2\left(\sigma_{j}=1\right)$ and $\pi / 2 \leq \psi_{B}^{*} \leq \pi\left(\sigma_{j}=-1\right)$ are called the face side and back side of sphere, respectively. Thus, $\sigma_{1}$ and $\sigma_{2}$ are valuated as follows

$$
\begin{aligned}
\text { upper sphere, face side }(\mathrm{F}) & \rightarrow \sigma_{1}=1 \\
\text { upper sphere, back side (B) } & \rightarrow \sigma_{1}=-1 \\
\text { lower sphere, face side (F) } & \rightarrow \sigma_{2}=1 \\
\text { lower sphere, back side (B) } & \rightarrow \sigma_{2}=-1 .
\end{aligned}
$$


Bearing in mind that $1+B^{2}-a$ in (26) may obtain both positive and negative values, the ranges of variation of $\phi_{B}$ may be specified if all restrictions on $a, B$ would be taken into account (see Table below).

\begin{tabular}{|l|c|c|c|c|}
\hline PR & Und & Und & Nod, $H>0$ & Nod, $H<0$ \\
\hline & $B^{2}<a-1<B$ & $-B<a-1<B^{2}$ & $B<a+1$ & $B<a+1$ \\
\hline F/B & \multicolumn{3}{|c|}{$\sigma_{2}=1$ or $\sigma_{1}=-1$} & $\sigma_{1}=1$ or $\sigma_{2}=-1$ \\
\hline$\phi_{B}$ & {$[0, \pi / 2] \pm 2 \pi$} & {$[\pi / 2, \pi] \pm 2 \pi$} & {$[\pi, 3 \pi / 2] \pm 2 \pi$} & {$[\pi / 2, \pi] \pm 2 \pi$} \\
\hline F/B & \multicolumn{3}{|c|}{$\sigma_{2}=-1$ or $\sigma_{1}=1$} & $\sigma_{2}=1$ or $\sigma_{1}=-1$ \\
\hline$\phi_{B}$ & {$[3 \pi / 2,2 \pi] \pm 2 \pi$} & {$[\pi, 3 \pi / 2] \pm 2 \pi$} & {$[\pi / 2, \pi] \pm 2 \pi$} & {$[\pi, 3 \pi / 2] \pm 2 \pi$} \\
\hline
\end{tabular}

A concave Nod $\left(S_{H}=-1\right)$ is considered separately. In $(20,21)$ the first formula in (20) is changed,

$$
\tan \psi_{B}^{*}= \pm \frac{1+B \cos \phi_{B}}{B \sin \phi_{B}}, \quad b=a
$$

where $-/+$ sign corresponds to the lower (upper) sphere. Keeping in mind that only the face sides of both spheres are permitted for concave Nod we arrive at the range of $\phi_{B}$ given in Table above, where a symbol $\left[\gamma_{1}, \gamma_{2}\right] \pm 2 \pi$ denotes three different ranges: $\left[\gamma_{1}, \gamma_{2}\right],\left[\gamma_{1}+2 \pi, \gamma_{2}+2 \pi\right]$ and $\left[\gamma_{1}-2 \pi, \gamma_{2}-2 \pi\right]$.

According to [3], Section 6.2, there exist the Und and Nod menisci with completely concave meridional profiles (without IP, see Fig. 1a) which are allowed for the F-F spheres arrangement. Such menisci do exist in the F-B arrangements if the spheres radii $a_{1}>a_{2}$ and menisci parameters $B$ satisfy

$$
\begin{aligned}
& \text { Nod: } \arccos \left(-\frac{1+B^{2}+a_{1}}{B\left(2+a_{1}\right)}\right)<\arccos \left(-\frac{1+B^{2}+a_{2}}{B\left(2+a_{2}\right)}\right) \\
& \text { Und : } \arccos \left(-\frac{1+B^{2}-a_{1}}{B\left(2-a_{1}\right)}\right)>\arccos \left(-\frac{1+B^{2}-a_{2}}{B\left(2-a_{2}\right)}\right) .
\end{aligned}
$$

According to (29) both concave menisci (Und and Nod) do not exist in the F-B arrangement if $a_{1}=a_{2}$. Finally, in case of the B-B spheres setup the existence of the concave menisci is forbidden.

\subsection{Constraint of C Type}

The conditions $(14,15)$ derived for the third case of meniscus nonexistence reduce to the following relations for $1<B<a_{i}+1$ in an assumption that the meniscus 
does not "pierce" the $i$-th SB

$$
\begin{aligned}
& M\left(S_{H} \phi_{1}, B\right)-M\left(S_{H} \phi_{2}, B\right)+\left[\sigma_{I} A_{i}\left(\phi_{I}\right)-A_{i}\left(\phi_{i}\right)\right]=0 \\
& A_{i}\left(\phi_{j}\right)=\sqrt{a_{I}^{2}-\left(1+B^{2}+2 B \cos \phi_{j}\right)}, \quad I=(i+1)(\bmod 2) .
\end{aligned}
$$

Coexistence of the $\mathbf{A}, \mathbf{B}$ and $\mathbf{C}$ types of constraints may be found in Fig. 6.

\subsection{Constraint of D Type}

Substitute $(5,17)$ into $(16)$ and obtain the condition of the proper SB positioning,

$$
M\left(S_{H} \phi_{1}, B\right)-M\left(S_{H} \phi_{2}, B\right)+\sigma_{1} A_{2}\left(\phi_{1}\right)+\sigma_{2} A_{1}\left(\phi_{2}\right)=0 .
$$

Coexistence of the A, B and $\mathbf{D}$ types of constraints may be found in Figs. 8c and 9c,d. In Fig. 3 we present two typical domains of menisci existence.

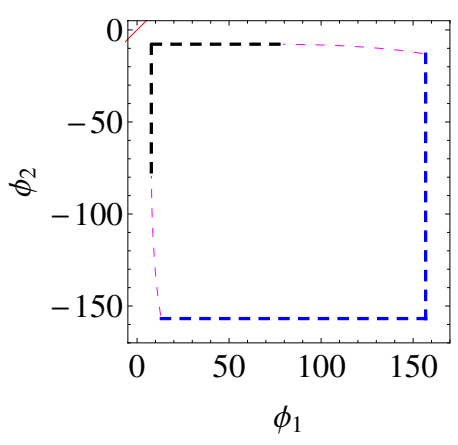

a)

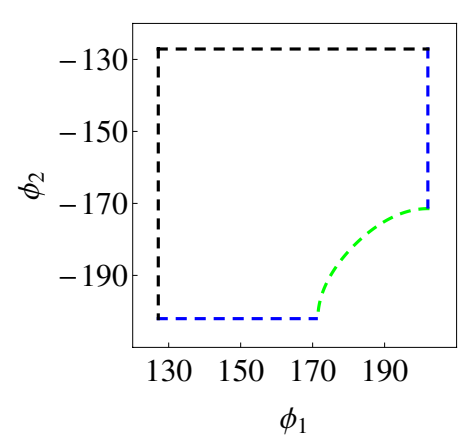

b)

Figure 3. (Color online) Coexistence of the A (black), B (blue), $\mathbf{C}$ (magenta) and $\mathbf{D}$ (green) types constraints for Nod between two equal spheres: a) F-F setup, $B=1.205, a=2.2$; b) B-B setup, $B=1.5, a=1.2$.

\section{Menisci Between Equal Spheres. Face-to-Face Setup}

We present a gallery of images showing for given value of $B$ in the plane $\left\{\phi_{1}, \phi_{2}\right\}$ the regions of existence (limited by the dashed curves) and inside them the regions of stability (shading shown in blue for $S_{H}=1$ and in light orange for $S_{H}=-1$ ). These images should not be understood as solution of the problem of meniscus existence between the two solid spheres at a given distance $d$ between their centers with prescribed contact angles $\theta_{i}$. On the contrary, a point $\left(\phi_{1}, \phi_{2}\right)$ in the region of existence determines an axisymmetric meniscus with a meridional profile given 
by (5) for $\phi_{2} \leq \phi \leq \phi_{1}$. This meniscus makes some contact angles $\theta_{i}$ with the solid spheres which can be computed using simple trigonometric relations, while the distance $d$ is computed from (3). If this point appears in the shaded area the corresponding meniscus is stable.

The red curves in Figures show the location of Stab domain boundary for the menisci with the fixed CL. The brown lines show the change in the number of IP in the meridional Und profile. The number of IPs in Und profile is denoted in red, e.g., $2^{+}$means two IPs on the meniscus meridional section $M$ which is convex in vicinity of $\phi=\phi_{1}$ and $1^{-}$means one IP on $M$ which is concave in vicinity of $\phi=\phi_{1}$. Four different types of meniscus existence boundaries are denoted in black $(\mathbf{A})$, blue $(\mathbf{B})$, magenta $(\mathbf{C})$ and green $(\mathbf{D})$ colors. In the first series of the images in Fig. 4, the coordinates $\phi_{1}, \phi_{2}$ are labelled, but further on they are dropped to improve the visual perception.

\subsection{Unduloidal Menisci Between Two Solid Spheres}

In this section we present the stability diagrams for Und menisci between two equal spheres. These diagrams were found by analyzing the positiveness of the matrix $Q_{i j}$ in (1). In Figs. 4 and 5a,b such diagrams are presented for a wide range of $B$. In the case $B=a-1$ we find another phenomenon: the boundaries of stability domains for fixed and free CL meet (this question was left open in [3]). In all cases there exist three kinds of stable Und menisci: without IPs and with one or two IPs.

Instability of Und menisci with more than one IP became a sort of folklore although there is no any rigorous claim in this regards. E.g., dealing with menisci between solid sphere contacting the plate the authors [10] posed a statement which was not supported by calculation: "There might be more than one IP ... . Multiple IPs in the meridional profiles are known but such menisci are likely to be unstable". Although in [3] we have shown that Und menisci with more than one IP between two solid parallel plates are always unstable, the general statement for two arbitrary SB remains elusive.

A strong statement about stability of axisymmetric menisci between two solid spheres has been announced in [17, Theorem on p.374], and its equivalent version in [18, p.397] reads: "the convex Und or Sph menisci are stable, while the convex Nod meniscus is unstable. The solid spheres have not to be equal or have equal contact angles". 


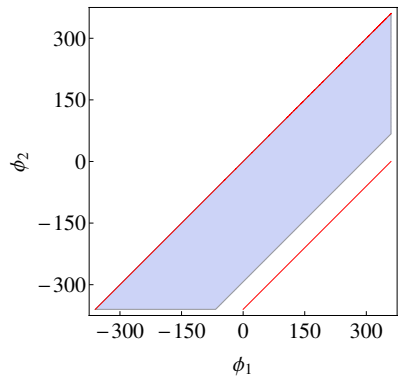

a)

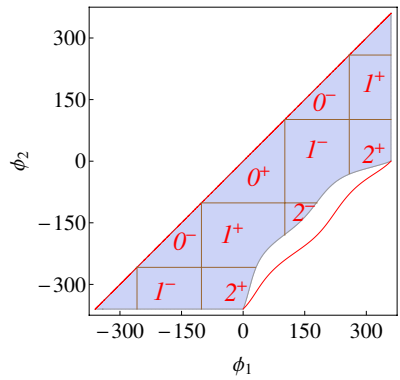

c)

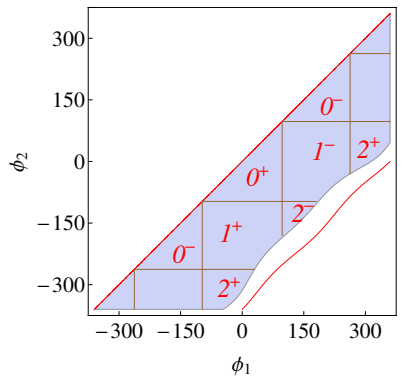

b)



d)

Figure 4. (Color online) Stability diagrams for F-F setup of a) Cyl meniscus, $B=0$, and three Und menisci, b) $B=0.15$, c) $B=0.2$ and d) $B=0.25$, between two solid spheres of radius $a=1.2$. The number of IPs in Und profile is denoted in red throughout the whole manuscript.

\subsection{Nodoidal Menisci Between Solid Spheres (2 Types of Constraints)}

Considering the Nod menisci it should be underlined that part of the plane $\left\{\phi_{1}, \phi_{2}\right\}$ where the meniscus with $S_{H}=-1$ exists is determined by relation (6) and the existence conditions. The Stab domain (shown in light orange) covers either a part of (Fig. 5c) or the whole Exst (Fig. 5d). At the same time the convex Nod menisci with $S_{H}=1$ for $1<a<2$ appear to be stable everywhere they exist (Fig. 5c,d).

\subsection{Nodoidal Menisci Between Solid Spheres (3 Types of Constraints)}

For some parameter values one can observe a special case when Exst domain is bounded by three types of constraint. Such an example is illustrated in Fig. 6 where Exst and Stab regions for the Nod menisci are shown. Note that the concave Nod meniscus for $B=1.05$ is unstable in small part of Exst, while for larger values of $B$ these menisci are stable everywhere in the corresponding Exst region. 


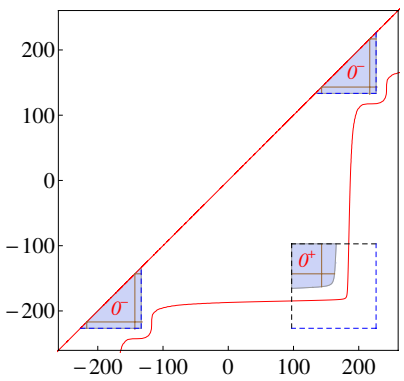

a)

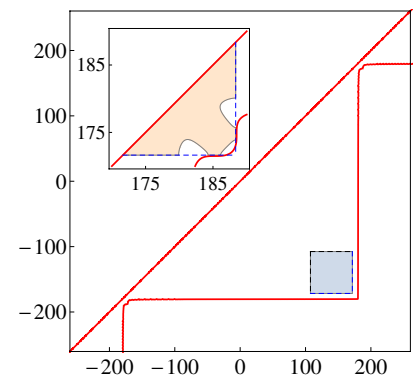

c)

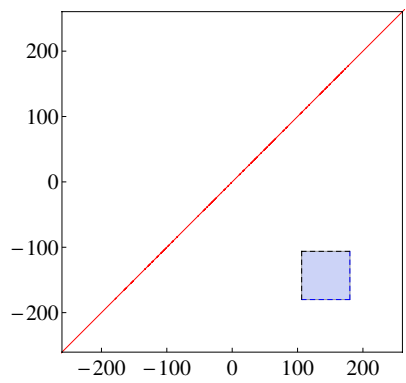

b)

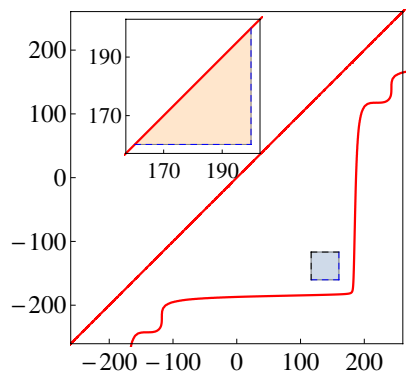

d)

Figure 5. (Color online) Stability diagrams for F-F setup of a) Und meniscus, $B=0.8$, b) Sph meniscus, $B=1$, and two Nod menisci, c) $B=1.03$, d) $B=1.25$, between two solid spheres of radius $a=1.2$.

\subsection{Menisci Between Two Equal Contacting Spheres}

In this section we analyze a special case of liquid bridges between two equal contacting spheres to check recent claims made in [19]. For convenience we make use of menisci classification given independently in [11] and [18]. Following formulas $(6,7)$ in [11] define $\alpha$ as a real root of the equation

$$
1+4 \alpha(\alpha-1) \sin ^{2}(\theta+\psi)=B^{2}, \quad \theta=\theta_{1}=\theta_{2}, \quad \psi=\psi_{1}=\psi_{2} .
$$

\begin{tabular}{|c|c|c|c|c|c|c|c|}
\hline & Nod $^{-}$ & Cat & Und & Cyl & Und & Sph & Nod $^{+}$ \\
\hline \hline$\alpha$ & $<0$ & 0 & $(0,1 / 2)$ & $1 / 2$ & $(1 / 2,1)$ & 1 & $>1$ \\
\hline$B$ & $>1$ & - & $(0,1)$ & 0 & $(0,1)$ & 1 & $>1$ \\
\hline
\end{tabular}

This produces a correspondence $\alpha \leftrightarrow B$ (excluding the Cat meniscus). The only difference with [18] is that it used $A=-\alpha$, where Cyl occurs only if $\theta+\psi=\pi / 2$ 


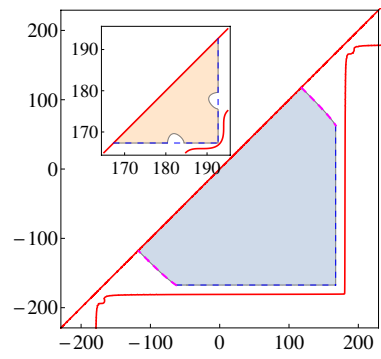

a)

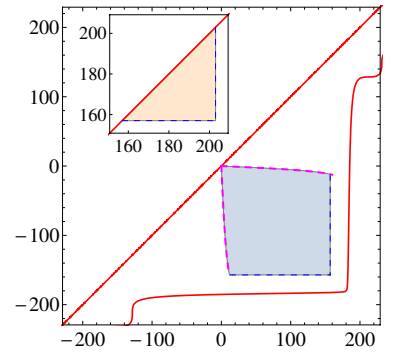

b)

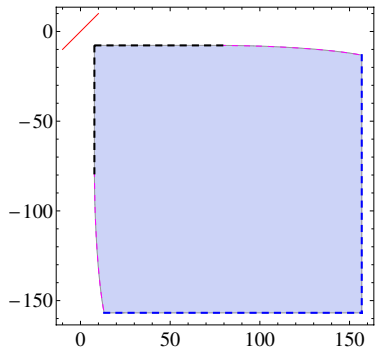

c)

Figure 6. (Color online) Stability diagrams for F-F setup of Nod menisci a) $B=1.05$, b) $B=1.2$, c) $B=1.205$ between two solid spheres of radius $a=2.2$. In Fig. $6 \mathrm{c}$ we focus on that part of stability domain which corresponds to the convex Nod: its boundaries comprise all three types of constraints.

and $\operatorname{Nod}^{ \pm}$denote the nodoid menisci with negative (-) or positive (+) curvature $H$, respectively. A sequence of menisci listed in Table is presented in [11, Fig. 6]. The following statements about existence of axisymmetric menisci between two equal contacting spheres have been announced in [19]:

Theorems 3.3 and Theorem 3.4. "For $\pi / 2<\theta<\pi$ and $\alpha<0\left[\mathrm{Nod}^{-}\right]$and $\pi / 2 \leq$ $\theta<\pi$ and $\alpha<1\left[\mathrm{Nod}^{-}\right.$, Cat, Und, Cyl], no liquid bridge between contacting balls exists which is both axisymmetric and symmetric across the plane which is the perpendicular bisector of the line segment between the centers of the balls."

Note 3.5. "For $\alpha>1\left[\mathrm{Nod}^{+}\right]$, there may be axisymmetric bridges between contacting balls, but these are known to be unstable [17]. There do not exist stable axisymmetric bridges between contacting balls with: a) $\theta \geq \pi / 2$, b) rotation symmetry, c) symmetry across the perpendicular bisector of the line segment between the centers of the balls."

Consider the case when the meniscus has a contact angle with the sphere equal to $\pi / 2$ and two spheres contact each other. The inclination angle $\alpha$ with the plane of the meniscus tangent at the contact point can be expressed through the similar angle $\psi$ of the tangent to the sphere as follows: $\psi=\alpha \pm \pi / 2$, where the lower (upper) sign is chosen for $0 \leq \psi \leq \pi / 2(\pi / 2 \leq \psi \leq \pi)$. At the same time we have

$$
-\frac{1+B \cos \phi}{B \sin \phi}=\tan \alpha, \frac{a \sin \psi}{\sqrt{1+B^{2}+2 B \cos \phi}}=1, \quad a-a \cos \psi=M(\phi, B)
$$


where the last two equations determine the conditions $r(\phi)=R(\psi), z(\phi)=$ $Z(\psi)$ at the contact point. These equations produce

$$
a=\frac{r^{2}(\phi)+M^{2}(\phi, B)}{2 M(\phi, B)}, \quad \tan \psi=\frac{2 r(\phi) M(\phi, B)}{r^{2}(\phi)-M^{2}(\phi, B)} .
$$

Using the relation $\tan \psi=-\cot \alpha$ from the first equation in (33) we find

$$
2(1+B \cos \phi) r(\phi) M(\phi, B)=\left(r^{2}(\phi)-M^{2}(\phi, B)\right) B \sin \phi
$$

which allows to find for given $B$ the coordinate $\phi$ of the contact point, and the sphere of radius $a$.

A setup of meniscus between two contacting equal spheres poses a question about a relation between $\phi_{1}$ and $\phi_{2}$. The contact points on the spheres has the coordinates

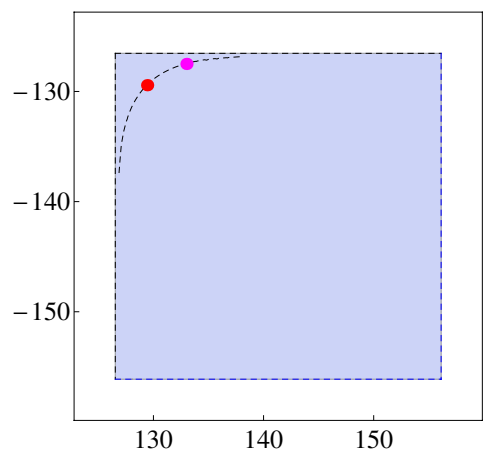

Figure 7. (Color online) A stable domain for Nod menisci $(B=2.15)$ with F-F setup between two equal touching spheres of radius $a=1.75$ and two menisci: with (red point) and without (magenta point) symmetry across perpendicular bisector of the line segment between the centers of the balls. Contact angles of both menisci on spheres are $\theta>\pi / 2$.

satisfying the relations

$$
\begin{aligned}
& r_{i}=a \sin \psi_{i}=\sqrt{1+B^{2}+2 B \cos \phi_{i}} \\
& z_{1}=M\left(\phi_{1}\right)=d_{1}-a \cos \psi_{1}, \quad z_{2}=M\left(\phi_{2}\right)=d_{2}+a \cos \psi_{2}
\end{aligned}
$$

where $d_{i}$ denotes the position of the $i$-th sphere center on the vertical axes, so that for the contacting spheres we have $d_{1}-a=d_{2}+a$, or $d_{1}-d_{2}=2 a$. The last equality leads to the desired relation

$$
\begin{aligned}
M\left(\phi_{1}\right)-M\left(\phi_{2}\right)= & 2 a-\sqrt{a^{2}-\left(1+B^{2}+2 B \cos \phi_{1}\right)} \\
& -\sqrt{a^{2}-\left(1+B^{2}+2 B \cos \phi_{2}\right)} .
\end{aligned}
$$


In Fig. 7 we present the stability diagram for Nod meniscus and label by red and magenta points (belonging to the gray curve defined by (36)) the location of stable menisci between two equal contacting spheres with contact angle $\theta>\pi / 2$. This refutes the statement Note 3.5 in [19] in both cases: with and without symmetry across perpendicular bisector of the line segment between the centers of the spheres.

\section{Menisci Between Equal Spheres. Back-to-Back Setup}

The stability analysis in this case is performed similarly to the case of F-F setup, but the sequence and structure of Stab with increasing value of $B$ appears to be much simpler. One of the reasons of such simplification is that the Nod meniscus with negative curvature is forbidden in this setup. To illustrate this point we consider three characteristic ranges of values of the solid sphere radius: $a<1,1<a<2$, and $a>2$.

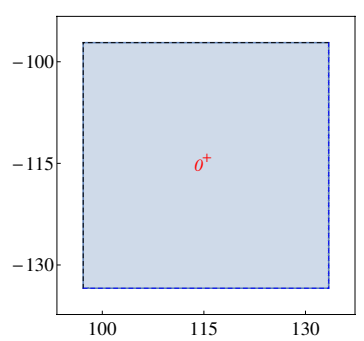

a)

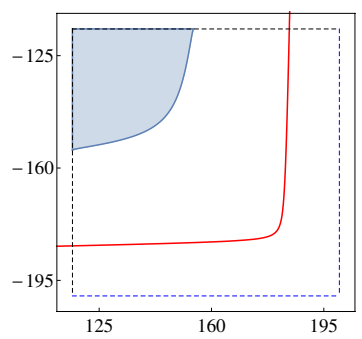

b)

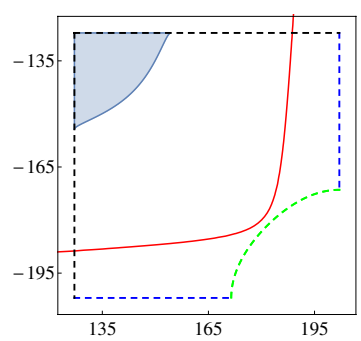

c)

Figure 8. (Color online) Stability diagrams for B-B setup of a) Und meniscus, $B=0.8$, and two Nod menisci, b) $B=1.25$, c) $B=1.5$, between two solid spheres of radius $a=1.2$.

First consider the case $1<a<2$, choosing $a=1.2$; the computation shows that the Und meniscus has no IPs and is stable everywhere it exists (Fig. 8a). The Nod meniscus is stable in smaller part of the existence region Exst which boundary may be determined by the existence condition $\mathbf{D}$ (see Figs. 8b,c).

In the case $a<1$ we observe that the Und meniscus has two IPs and again is stable everywhere it exists (Fig. 9a); the same time Stab region of the convex Nod meniscus covers only some part of Exst (Fig. 9b). Finally, when $a>2$ the existence region of the Nod meniscus is strongly limited by the existence condition D and these menisci are stable in the large part of Exst (see Fig. 9c,d). 




a)

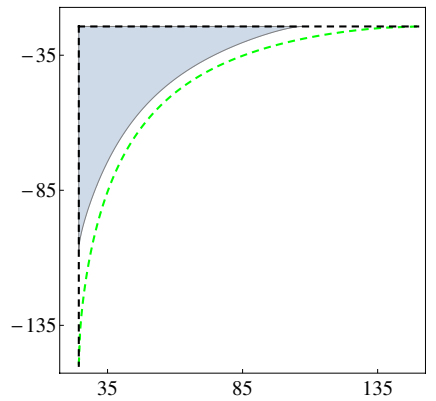

c)

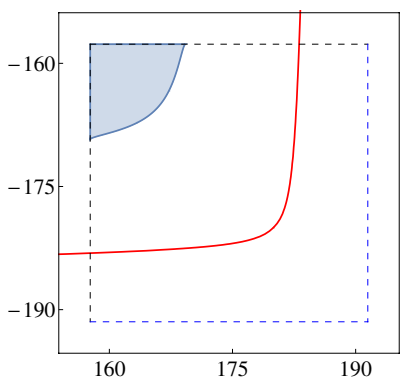

b)

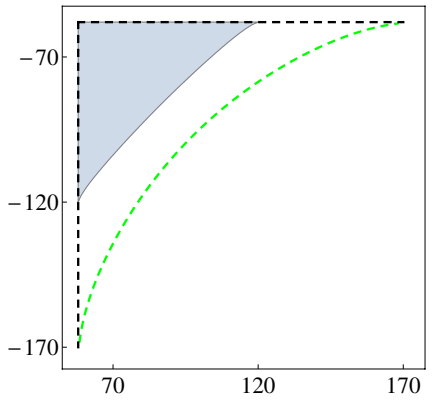

d)

Figure 9. (Color online) Stability diagrams for B-B setup of a) Und meniscus, $B=0.8$, and b) Nod menisci, $B=1.25$, between two solid spheres of radius $a=0.5$, c) $B=1.25$, and (d) $B=1.5$, between two solid spheres of radius $a=2.2$.

\section{Menisci Between Equal Spheres. Face-to-Back Setup}

The F-B setup is quite simple for the analysis, as in this case the boundaries of Exst can be described as a "outer product" of the corresponding regions for F-F and B$\mathrm{B}$ setups. To explain this feature consider the case when the meniscus touches the face of the upper SB at $\phi=\phi_{1}$, and the back of the lower SB at $\phi=\phi_{2}$. The existence conditions $\mathbf{A}$ and $\mathbf{B}$ (represented by the black and blue broken lines) are determined for $\phi_{1}$ and $\phi_{2}$ independently. It is illustrated for Und menisci in Figs. 10a,b where the range of the accessible values for $\phi_{1}$ is much larger than for $\phi_{2}$. For Nod menisci (see Figs. 10c,d) the range of the accessible values for $\phi_{1}$ and $\phi_{2}$ is comparable. 


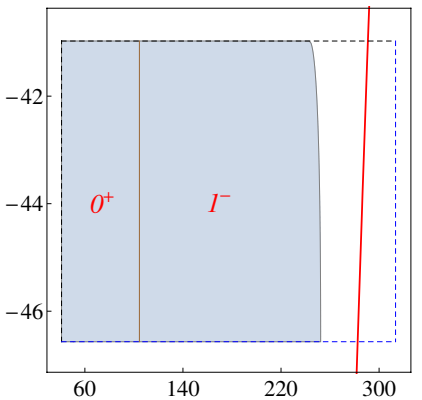

a)

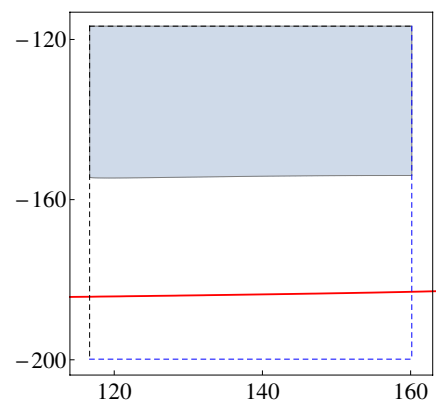

c)

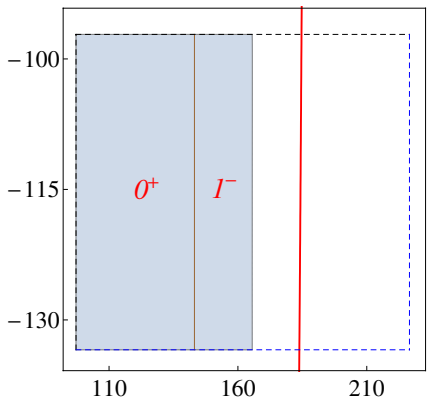

b)

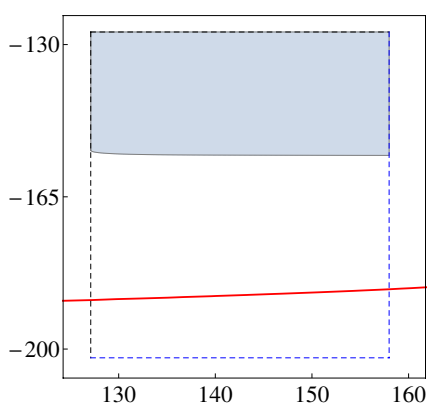

d)

Figure 10. (Color online) Stability diagrams for F-B setup of Und menisci, a) $B=0.25$ and b) $B=0.8$, and Nod menisci, c) $B=1.25$ and d) $B=1.5$, between two solid spheres of radius $a=1.2$.

\section{Menisci Between Nonequal Spheres}

The existence and stability analysis in the case of solid spheres of unequal radii is similar to the case of F-B setup considered in Section 6. The boundaries of Exst determined by the conditions $\mathbf{A}$ and $\mathbf{B}$ depend on the corresponding sphere radii and have to be computed independently. This breaks the symmetry of Exst and Stab w.r.t. the line $\phi_{1}+\phi_{2}=0$. A difference in spheres radii may lead to existence of special types of menisci which are forbidden in setup with equal radii.

\subsection{Face-to-Face Setup}

In Fig. 11 we present the stability diagrams for Und and Nod menisci for $1<a_{1}<$ 2 and $a_{2}>2$. By comparison to Figs. $4 \mathrm{~d}$ and $11 \mathrm{a}, 5 \mathrm{a}$ and $11 \mathrm{~b}, 6 \mathrm{c}$ and $11 \mathrm{c}$, one may see how the stability diagrams become asymmetric w.r.t. the line $\phi_{1}+\phi_{2}=0$. 




a)

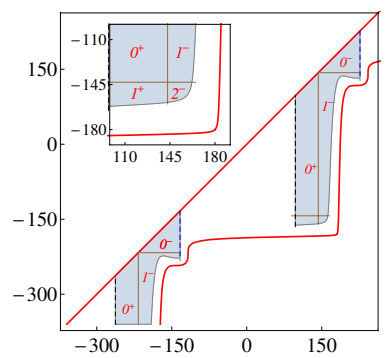

b)



c)

Figure 11. (Color online) Stability diagrams for F-F setup of Und menisci, a) $B=0.25$, b) $B=0.8$, and Nod meniscus, c) $B=1.205$, between two nonequal solid spheres of radii $a_{1}=1.2$ and $a_{2}=2.2$.

\subsection{Face-to-Back Setup}

The F-B setup of menisci between two nonequal spheres gives rise to existence of concave Nod meniscus which is forbidden in F-B setup between two equal spheres (see Fig. 11).

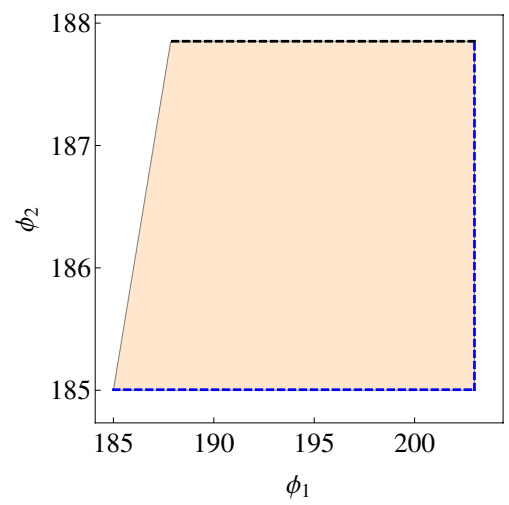

a)

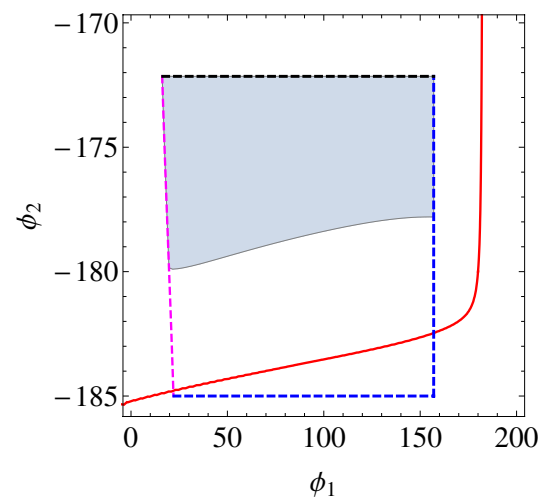

b)

Figure 12. (Color online) Stability diagrams for F-B setup of concave a) and convex b) Nod menisci, $B=1.2$, between two non equal solid spheres of radii $a_{1}=2.2$ and $a_{2}=0.25$. A red line in Fig. 12a is a main diagonal in the plane $\left\{\phi_{1}, \phi_{2}\right\}$. A magenta curve in Fig. 12b describes the $\mathbf{C}$ constraint of existence.

The trapezoidal geometry of Exst in Fig. 12a appears due to intersection of triangular existence region for concave Nod meniscus in the F-F setup between two 
equal spheres of radius $a_{1}$ with existence constraint $\mathbf{A}$ on sphere of radius $a_{2}<a_{1}$ that results in the triangle cut. Note that for the parameters selected in Fig. 12a the concave Nod meniscus is stable in every point of Exst. In case of the convex Nod meniscus a part of the boundaries of Exst may be related to the $\mathbf{C}$ type constraint (see Fig. 12b).

\section{Acknowledgement}

The research of LF was supported in part by the Kamea Fellowship.

\section{References}

[1] Bostwick J. and Steen P., Dynamics of Sessile Drops. Part 1. Inviscid Theory, J. Fluid Mech. 760 (2014) 5-38.

[2] Delaunay C., Sur la surface de révolution dont la courbure moyenne est constante, J. Math. Pure et Appl. 16 (1841) 309-315.

[3] Fel L. and Rubinstein B., Stability of Axisymmetric Liquid Bridges, Z. Angew. Math. Phys. (2015) 25 pp., http://link.springer.com/article/10.1007/s00033015-0555-5

[4] Finn R. and Vogel T., On the Volume Infimum for Liquid Bridges, Z. Anal. Anwend. 11 (1992) 3-23.

[5] Hadzhilazova M., Mladenov I. and Oprea J., Unduloids and their Geometry, Archivum Mathematicum 43 (2007) 417-429.

[6] Kenmotsu K., Surfaces of Revolution with Prescribed Mean Curvature, Tohoku Math. J. 32 (1980) 147-153.

[7] Kilian, M., Rossman W. and Schmitt N., Delaunay ends of Constant Mean Curvature Surfaces, Compos. Math. 144 (2008) 186-220.

[8] Mladenov I., Conformal Immersions of Delaunay Surfaces and Their Duals, Geometry, Integrability \& Quantization 5 (2004) 158-168.

[9] Myshkis A., Babskii V., Kopachevskii N., Slobozhanin L. and Tyuptsov A., Low-Gravity Fluid Mechanics, Springer, New York 1987.

[10] Orr F., Scriven L. and Rivas A., Pendular Rings Between Solids: Meniscus Properties and Capillary Forces, J. Fluid Mech. 67 (1975) 723-744.

[11] Rubinstein B. and Fel L., Theory of Axisymmetric Pendular Rings, J. Colloid Interf. Sci. 417 (2014) 37-50. 
[12] Strube D., Stability of Spherical and Catenoidal Liquid Bridge Between Two Parallel Plates in Absence of Gravity, Micrograv. Sci. Technol. 4 (1991) 263269.

[13] Sultana N., Explicit Parameterization of Delaunay Surfaces in Space Forms via Loop Group Methods, Kobe J. Math. 22 (2005) 71-107.

[14] Vogel T., Stability of a Liquid Drop Trapped Between Two Parallel Planes, SIAM J. Appl. Math. 47 (1987) 516-525.

[15] Vogel T., Stability of a Liquid Drop Trapped Between Two Parallel Planes II: General Contact Angles, SIAM J. Appl. Math. 49 (1989) 1009-1028.

[16] Vogel T., Non-Linear Stability of a Certain Capillary Problem, Dynamics of Continuous, Discrete and Impulsive Systems 5 (1999) 1-16.

[17] Vogel T., Convex, Rotationally Symmetric Liquid Bridges Between Spheres, Pacific J. Math. 224 (2006) 367-377.

[18] Vogel T., Liquid Bridges Between Balls: The Small Volume Instability, J. Math. Fluid Mech. 15 (2013) 397-413.

[19] Vogel T., Liquid Bridges Between Contacting Balls, J. Math. Fluid Mech. 16 (2014) 737-744.

[20] Wente H., The Symmetry of Sessile and Pendent Drops, Pacific J. Math. 88 (1980) 387-397.

[21] Zhou L., On Stability of a Catenoidal Liquid Bridge, Pacific J. Math. 178 (1997) 185-198.

Received 03 July 2015

Boris Y. Rubinstein

Stowers Institute for Medical Research

1000 E 50th St, Kansas City, MO 64110, USA

E-mail address: bruestowers . org

Leonid G. Fel

Department of Civil Engineering

Technion - Israel Institute of Technology

Haifa, 32000, Israel

E-mail address: Ifeletechnion.ac.il 